\title{
Jet Physics in ep Collisions
}

\author{
Valentin Efremenko, on behalf of the H1 and ZEUS collaborations \\ Institute for Theoretical and Experimental Physics, Moscow, Russia
}

Received on 01 November, 2006; Revised on 30 January, 2007

\begin{abstract}
The most recent results of jet cross section measurements at HERA collider in photoproduction and deep inelastic scattering are presented. The forward jet and multiple jet productions are discussed from point of view of QCD dynamics. Results of precise extractions of the strong coupling $\alpha_{s}$ are presented.
\end{abstract}

Keywords: Jet physic; QCD hard interactions

During the last years a noticeable progress has been achieved in measuring and understanding of jet production at high energies. H1 and ZEUS are two multipurpose experiments, which are well suited for study of jet physics. Both experiments located in North and South halls of the HERA electron-proton collider in Hamburg, Germany, solve the same problem using different methods.

Both experiments are designed using in principle the same lay-out. The interaction vertex is determined with central tracking detectors, which are surrounded by electromagnetic and hadronic calorimeters. The luminosity is measured from the rate of the bremsstrahlung process $e p \rightarrow e p \gamma$. Detail descriptions of the detectors can be found in $[1,2]$.

\section{PHOTOPRODUCTION OF DI-JETS}

The largest cross section at HERA collider is due to photoproduction process with the momentum transfer squared, $Q^{2}$, almost equal to zero. Nevertheless, the photoproduction of dijets with high transverse momenta can be calculated within perturbative Quantum Chromo Dynamic (QCD) where the transverse momentum of jets provides the hard scale.

The measured cross section of di-jet events in the photoproduction as a function of variables $x_{p}$ and $x_{\gamma}$ is shown in Fig. 1 [3]. In the leading order $x_{p}$ and $x_{\gamma}$ are the longitudinal photon and proton momentum fractions entering the hard interaction. The transverse energy of the first jet is more than $25 \mathrm{GeV}$ and the transverse energy of the second jet is more than $15 \mathrm{GeV}$.

Two top histograms are presented for the resolved enriched events $\left(x_{\gamma}<0.8\right)$, when the photon interacts through its hadronic contents, and for the direct events $\left(x_{\gamma}>0.8\right)$, when photon interacts as a point-like particle.

Two bottom histograms are from two subsamples of events for $x_{p}<0.1$ and $x_{p}>0.1$. For small $x_{p}$, about $70 \%$ of events are induced by gluons. This means that these two subsamples of events roughly distinguish between photon-gluon fusion $\left(x_{p}<0.1\right)$ and photon-quark scattering $\left(x_{p}>0.1\right)$.

The next to leading order (NLO) QCD calculation and the PYTHIA Monte Carlo (MC) calculation provide a reasonable descriptions of the data. These data, combined with inclusive deep inelastic scattering (DIS) cross section measurements, help to extract proton parton density function (PDF) with improved precision.
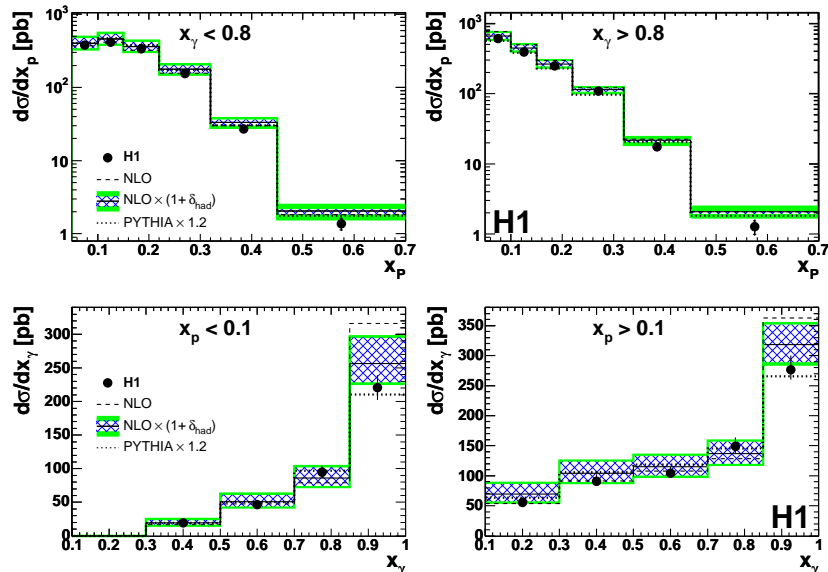

FIG. 1: Cross section as a function of $x_{\gamma}$ (for two regions in $x_{p}$ ) and $x_{p}$ (for two regions in $x_{\gamma}$ ) for data (points), NLO QCD predictions with (solid line) and without (dashed line) hadronisation corrections and for PYTHIA Monte Carlo predictions (dotted line). The hatched and shaded bands for $\operatorname{NLOx}\left(1+\delta_{\text {had }}\right)$ result in the scale and the total uncertainties respectively.

\section{JETS IN DIS}

Currently the perturbative calculations in QCD for values of the four momentum transfer squared, $Q^{2}$, larger than a few $\mathrm{GeV}^{2}$ are very reliable. The inclusive jet and di-jet analysis allows to constrain the gluon density in the proton, to extract the proton PDF, to extract value of $\alpha_{s}$ [4].

Due to good understanding of the detectors, $\mathrm{H} 1$ and ZEUS collaborations have measured inclusive jet and di-jet cross sections with high accuracy. Fig. 2 shows the cross section of di-jet production in DIS process as a function of $Q^{2}$ measured by ZEUS Collaboration. Di-jet cross section was measured with high precision in the kinematic region $125<Q^{2}<$ $5000 \mathrm{GeV}^{2}$ [5]. The figure also includes the NLO QCD calculations with renormalisation scale $\mu_{R}^{2}=Q^{2}+\bar{E}_{T}^{2}, \mu_{R}^{2}=Q^{2}$ and $\mu_{R}^{2}=\bar{E}_{T}^{2}$. The lower part of the figure shows the relative differences of the data with respect to NLO QCD calculations. The largest contributions to systematic uncertainty come from uncertainty in absolute energy scale $( \pm 10 \%)$ and the correction factor for detector effects $( \pm 8 \%)$. The largest contribution to theoretical uncertainties $( \pm 20 \%)$ comes from $\mu_{R}$. The theoretical uncertainties are dominated.

The precise measurement of inclusive jet production, di-jet 


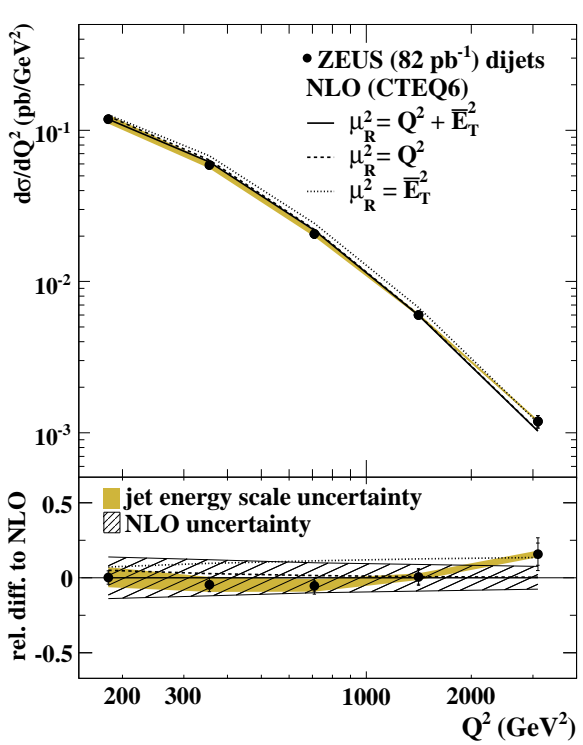

FIG. 2: The differential cross sections for di-jet production as a function of $Q^{2}$. The predictions of NLO QCD with different renormalisation scales are shown. The lower part of the figure shows the relative differences with respect to LO QCD calculations.

and three-jet production and reliable NLO calculations have allowed to extract the strong coupling constant $\alpha_{s}$ with the highest accuracy using different methods. The average extracted values of $\alpha_{s}$ at the Z-boson mass are

from H1: $\quad \alpha_{s}\left(M_{Z}\right)=0.1197 \pm 0.0016(\exp ) \pm 0.0047(t h)$; from ZEUS: $\alpha_{s}\left(M_{Z}\right)=0.1196 \pm 0.0025(\exp ) \pm 0.0023(t h)$. The world average value: $\alpha_{s}\left(M_{Z}\right)=0.1187 \pm 0.0020(\exp )$.

\section{JETS IN CHARGE CURRENT REACTIONS}

Since 2003 HERA collider operates with a longitudinally polarised lepton beams. The measurements of deep inelastic scattering with polarised lepton beams allow the parton distribution functions of the proton to be further constrained through polarisation asymmetries and specific tests of the electroweak part of the Standard Model to be performed. H1 and ZEUS collaborations have measured for the first time total and differential inclusive jet cross sections of the interaction of positrons and electrons with protons in charge current (CC) DIS reactions with polarised beams.

Figure 3 shows the CC negatively and positively polarised $e^{-} p$ DIS inclusive jet differential cross sections as a function of $Q^{2}$ [6]. The value of cross sections are changed by three order, but the behaviour of cross section shows a less rapid fall-off than what is observed in neutral current DIS processes due to the massive $\mathrm{W}$ propagator. For comparison, the predictions of the leading-logarithm parton shower MC ColourDipole Model is included in the figure. In the lower part of the figure the ratio of the cross sections for negatively and positively polarised electron beams is shown. It is in agreement with the measured polarisation ratio, $\left(1-P^{n e g}\right) /\left(1-P^{\text {pos }}\right)=$ $1.90 \pm 0.07$. The total jet cross sections in the studied kine-

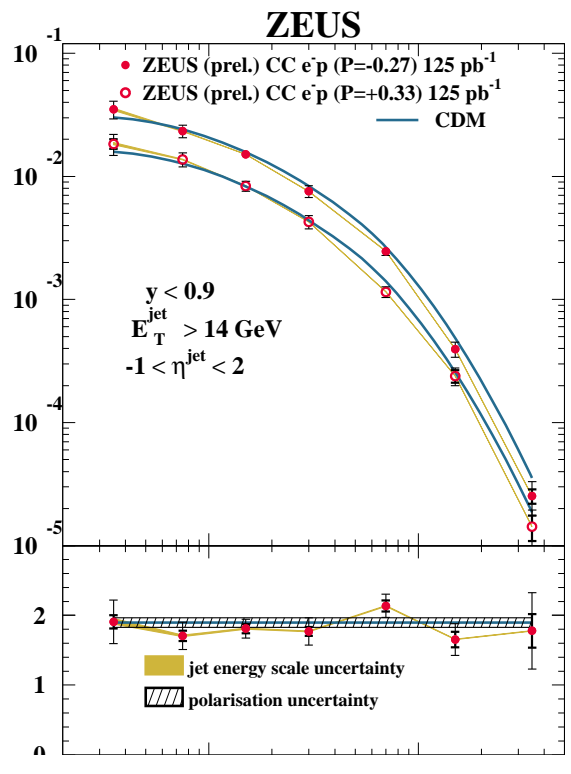

FIG. 3: The differential cross sections for $\mathrm{CC}$ inclusive jet events as a function of $Q^{2}$ for negative and positive polarised electron beams. The predictions of CDM model are included. The lower part of the picture displays the ratio of the cross sections for negatively and positive polarised electron beams.

matic region are in agreement with the MC predictions.

\section{FORWARD JETS}

QCD calculations based on DGLAP evolution scheme are successful in describing strong rise of $F_{2}\left(x_{B j}, Q^{2}\right)$ with decreasing Bjorken scaling variable $x_{B j}$ over a large $Q^{2}$ range. DGLAP scheme resums leading $\log \left(Q^{2}\right)$ terms and implies strong ordering of the transverse momenta of the emitted partons $k_{t}$.

Are there regions with a different parton dynamics?

The DGLAP approximation may become inadequate for small $x_{B j}$, where $\log (1 / x)$ terms become important in the evolution equation. Significant deviations from the LO DGLAP approach are observed in the forward jet production, transverse energy flow, fractional rate of di-jet production. Other approaches, like the BFKL, suggest different parton dynamics, which can be responsible for the observed deviation.

Let us consider a sample of events with a forward jet with pseudorapidity $\eta_{f w d j e t}>1.7$ and two additional jets with pseudorapidities $\eta_{\text {jet } 1}$ and $\eta_{\text {jet } 2}$ respectively. For $\eta_{f w j e t}>$ $\eta_{\text {jet } 2}>\eta_{\text {jet } 1}$ the differences between the pseudorapidities are $\Delta \eta_{1}=\eta_{j e t 2}-\eta_{j e t 1}$ and $\Delta \eta_{2}=\eta_{f w j e t}-\eta_{j e t 2}$.

In Fig. 4, the cross section for this sample of events as a function of $\Delta \eta_{2}$ is shown for $\Delta \eta_{1}<1$, for $\Delta \eta_{1}>1$ and for the total sample [7]. The result of NLO calculation from NLO$\mathrm{JET}++$ with hadronic correction is also shown with theoretical error included as bands.

If di-jet system originates from hard process and both additional jets are emitted in the central region, $\Delta \eta_{2}$ is large and 


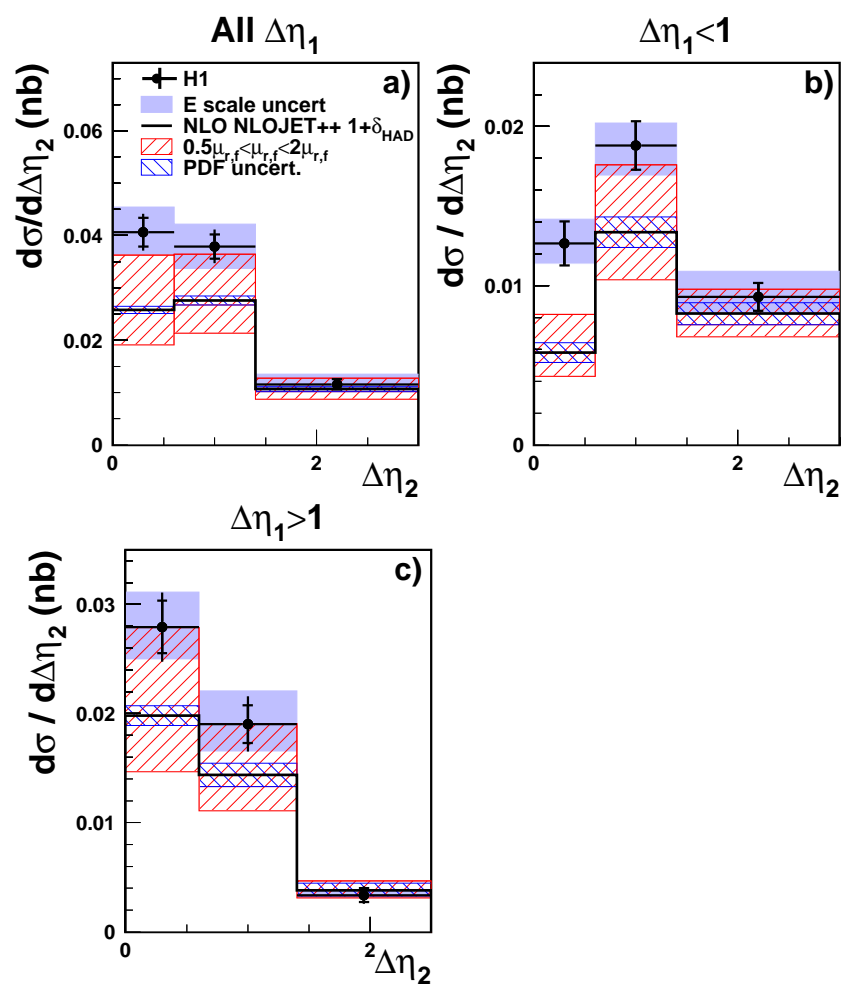

FIG. 4: The cross section for events with di-jet and forward jet as a function of the rapidity separation between the forward jet and the most forward-going additional jet. The data are compared to the predictions of NLOJET++.
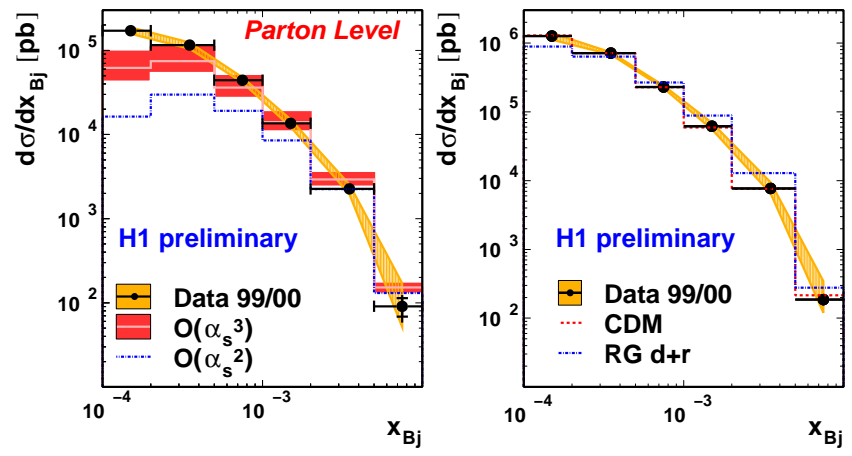

FIG. 5: Experimental (dots) differential cross sections as a function of the Bjorken scaling variable $x_{B j}$ for events with two forward jets. Data are compared to the LO $\left(\alpha_{s}^{2}\right)$ and NLO $\left(\alpha_{s}^{3}\right)$ QCD calculations (left picture) and to the predictions of CDM and RAPGAP with resolved photon (right picture).

the data are in agreement with NLOJET++ prediction. If the additional hard jets are shifted to the forward region and $\Delta \eta_{2}$ decreases, the agreement is not as strong. If both additional jets are in the forward region, $\Delta \eta_{1}$ and $\Delta \eta_{2}$ are small and with higher probability, all three jets are produced from gluon radiation. Because all three jets have transverse momenta larger than $6 \mathrm{GeV}$ (selection criteria) the strong $k_{t}$ ordering which is important for DGLAP evolution, does not work and NLO$\mathrm{JET}++$ prediction is much lower in comparison to the experimental data.

In Fig. 5 (left) the cross section of event production with two jets in the forward region as function of $x_{B j}$ is shown. The data are compared to the calculations predicted by LO $\left(\alpha_{s}^{2}\right)$ and NLO $\left(\alpha_{s}^{3}\right)$ QCD. The LO prediction does not describe the experimental data. Main discrepancy is seen at low $x_{B j}$. The prediction of NLO $\left(\alpha_{s}^{3}\right)$ QCD is much better, however more than $30 \%$ of events are still missing.

In the Colour Dipole Model, partons are generated by colour dipoles. Since the dipoles radiate independently there is no ordering in the transverse momenta of the emissions, which is similar to the BFKL approach. The RAPGAP MC program uses LO matrix element supplemented with initial and final state parton showers generated according to the DGLAP evolution. However in case of resolved process the photon interacts with proton through its hadron structure. This means that two evolution ladders are introduced, one from photon side and one from proton side and the strong $k_{t}$ ordering does not exist.

In Fig. 5(right) the cross section as function of $x_{B j}$ is shown for DIS production of three-jet events with two jets in the forward region. The experimental data are compared to LO Monte Carlo program DJANGO with Colour Dipole Model (CDM) and RAPGAP with resolved photon contribution. In both programs non $k_{t}$-ordering gluon radiation is implemented. The agreement with experimental data is remarkable.

\section{CONCLUSION}

Considerable progress in measurements and understanding of jet production is achieved at HERA as well as in theoretical calculations and reliable predictions. The good understanding of $\mathrm{pQCD}$ and precise measurements of the cross sections have allowed to extract one of the important parameters of theory $\alpha_{s}$ with an excellent accuracy. In some regions, however, theoretical uncertainties are dominated by scale variation effects.
[1] H1 Collaboration, I. Abt et al., Nucl. Instrum. Meth. A 386, 310 348 (1997).

[2] U. Holm (ed.) The ZEUS Detector, DESY (1993), available on http://www-zeus.desy.de/bluebook/bluebook.html

[3] H1 Collaboration, A. Aktas et al. Phys. Lett. B 639, 21 (2006).

[4] C. Glasman, hep-ex/0506035 (2005).

[5] ZEUS Collaboration, S. Chekanov et al. Preprint DESY-06-128,
DESY, 2006.

[6] ZEUS Collaboration, ZEUS-prel-06-002, ICHEP06 (2006), Moscow, Russia, avalable on http://www-zeus.desy.de/physics/ phch/conf/ichep06/

[7] H1 Collaboration, A. Aktas et al. Eur. Phys. J. C 46, 27 (2006). 